\title{
Violência conjugal: mapeamento do fenômeno no Rio Grande do Sul
}

\author{
Denise Falcke \\ Universidade do Vale do Rio dos Sinos \\ Mariana Gonçalves Boeckel \\ Universidade Federal de Ciências da Saúde de Porto Alegre \\ Adriana Wagner \\ Universidade Federal do Rio Grande do Sul
}

\begin{abstract}
Resumo
Os dados sobre violência conjugal costumam ser imprecisos devido a diversos fatores. Entre eles, o fato de retratarem, preponderantemente, a violência visível (física ou sexual), serem obtidos em delegacias e basearem-se na visão de apenas um dos parceiros; sendo que muitos casos não são denunciados. O objetivo deste estudo foi mapear a violência conjugal no Rio Grande do Sul, em relação à presença de coerção sexual, agressão física e psicológica. Participaram 751 casais de distintas regiões do Estado. Os instrumentos foram: questionário de dados sociodemográficos e Revised Conflict Tactics Scales (CTS2). Os resultados evidenciaram índices elevados de violência mútua e simétrica entre os casais, ou seja, cometida por ambos os parceiros. Identificou-se que a idade, a escolaridade, a renda e o tempo de relacionamento estiveram associados aos níveis de violência conjugal em suas diferentes dimensões $(p<0,05)$. Ressalta-se a necessidade de se repensar as intervenções com casais em situação de violência.

Palavras-chave: violência; relações conjugais; agressões sexuais; violência psicológica.
\end{abstract}

\section{Intimate partner violence: mapping the phenomenon in Rio Grande do Sul}

\begin{abstract}
Data on intimate partner violence are often inaccurate due to several factors. Among them, there are the facts that they portray mainly the visible violence (physical or sexual), are obtained in police stations and are based on the view of only one of the partners; considering that many cases are not reported. The aim of this study was to map domestic violence in the state of Rio Grande do $\mathrm{Sul}$, in relation to the presence of sexual coercion, physical and psychological aggression. 751 couples from different geographical areas of the state participated. The instruments used were: a socio-demographic questionnaire and Revised Conflict Tactics Scales (CTS2). Level of education, income, length and situation of the relationship were associated with conjugal violence in its different dimensions $(p<0,05)$. The results highlighted the existence of mutual and symmetrical aggressions between couples, differently from the traditional gender studies, pointing the importance to rethink the interventions with couples in situations of violence.
\end{abstract}

Keywords: violence; marital relations; sex offenses; psychological violence.

\section{La violencia conyugal: mapeo del fenómeno en Rio Grande do Sul}

\section{Resumen}

Los datos sobre la violencia de la pareja son a menudo inexactos debido a varios factores. Entre ellos, el hecho de retratar principalmente a la violencia visible (física o sexual), seren obtenidos en las comisarías y considerar la opinión de uno solo de los cónyuges. También demasiados casos no se notifican. El objetivo de este estudio fue mapear la violencia doméstica en Rio Grande do Sul, por la presencia de la coerción sexual, violencia física y agresión psicológica. Participaron 751 parejas de diferentes regiones del estado. Los instrumentos fueran cuestionario de datos sociodemográficos y Revised Conflict Tactics Scales (CTS2). Los resultados mostraron altos índices de violencia cometidos por los cónyuges. Se encontró que la edad, la educación, los ingresos y la duración de la relación se asociaron con niveles de violencia doméstica en sus distintas dimensiones $(p<0,05)$. Se hace hincapié en la necesidad de replantear las intervenciones con las parejas en situación de violencia.

Palabras clave: violencia; relaciones conyugales; delitos sexuales; violencia psicológica. 


\section{Introdução}

A violência conjugal é um fenômeno sociohistórico (Lourenço et al., 2013) que, por longo tempo, permaneceu sendo tratado como uma questão de foro íntimo, restrito ao ambiente familiar. Somente nas últimas décadas é que passou a ser entendido como um problema de saúde pública (Colossi, Razera, Haack, \& Falcke, 2015) e gerador de uma série de violações de direitos humanos (Monteiro \& Zaluar, 2013). Para além dos graves danos emocionais e físicos, os elevados índices de mortalidade evidenciam a seriedade do problema (Waiselfisz, 2015).

Aproximadamente $38 \%$ do número total de homicídios femininos no mundo são decorrentes da violência conjugal (World Health Organization, 2013). Com índice de 4,8 homicídios por $100 \mathrm{mil}$ mulheres, o Brasil ocupa a 5a posição entre 83 países, estando atrás somente de El Salvador, Colômbia, Guatemala e Federação Russa (Waiselfisz, 2015). Mais especificamente em relação ao Estado do Rio Grande do Sul, o Relatório Lilás de 2014 aponta que 69,6\% dos feminicídios foram praticados pelos companheiros atuais ou pelos ex-companheiros (Linch, 2014).

No que tange à prevalência mundial da violência, estima-se que aproximadamente $30 \%$ das mulheres sofram violência conjugal ao longo da sua vida (World Health Organization, 2013). No entanto, ao analisar os dados sobre essa realidade, é necessário considerar que eles costumam ser subestimados, uma vez que a maioria deles decorre de casos denunciados ou que chegam aos serviços de atenção à saúde (Alvim \& Souza, 2005), enquanto muitos casos nunca chegam a ser denunciados (Falcke, Oliveira, Rosa \& Bentancur, 2009).

A realidade brasileira a respeito da violência conjugal e suas diferentes manifestações foi estudada por Schraiber et al. (2007), que entrevistaram mulheres da cidade de São Paulo e da Zona da Mata Pernambucana (ZMP) e identificaram a violência psicológica como a mais preponderante $(41,8 \% \mathrm{em}$ SP e $48,9 \%$ na ZMP), seguida da violência física (27,2\% em SP e 33,7\% na ZMP) e, por fim, a violência sexual (10,1\% em SP e 14,3\% na ZMP). Nesse estudo, a violência psicológica acompanhou $90 \%$ dos casos de violência física ou sexual. Quanto à ocorrência de violência exclusiva, a psicológica foi a que apresentou taxa maior de ocorrência, aparecendo em 37,6\% dos casos em São Paulo e 32\% na ZMP (Schraiber et al., 2007).

A violência psicológica também foi observada em maior prevalência (55\%) dentre os resultados da pesquisa com usuárias de uma Unidade Básica de Saúde no Sul do Brasil (Kronbauer \& Meneghel, 2005). Em seguida, foi identificada a violência física $(38 \%)$ e, por último, a violência sexual $(9 \%)$. A maioria das mulheres relatou sofrer mais de um tipo de violência, sendo que $52 \%$ sofriam dois tipos de violência associados e $12 \%$ relataram sofrer os três tipos de violência concomitantemente. Gomes et al. (2012) apontam que, em geral, a violência psicológica acompanha as demais formas de manifestação de violência.

Quanto a violência física, Leôncio et al. (2008) a identificaram em $44,84 \%$ dos boletins de ocorrência registrados em outubro e novembro de 2007 na Delegacia de Defesa da Mulher, em Ribeirão Preto/SP, seguida pela violência verbal $(42,15 \%)$, pela violência psicológica $(8,97 \%)$ e pela violência sexual $(2,69 \%)$. A residência foi o principal local de ocorrência da violência (76\%).

Dados nacionais e internacionais evidenciam de forma clara a violência contra as mulheres (Waiselfisz, 2015; World Health Organization, 2013). Não há dúvidas da elevada prevalência e do impacto das relações de gênero acerca das mulheres vítimas, quando comparadas aos homens. Muitos estudos descrevem grandes contribuições acerca da cultura sexista e da importância de repensar as relações estabelecidas entre homens e mulheres, especialmente na esfera privada do lar. Entretanto, para além da mulher vítima, existem casos em que os homens também são vítimas de violência conjugal (Carmo et al., 2011; Drijber, Reijnders, \& Ceelen, 2013; Lovestad \& Krantz, 2012). Nos Estados Unidos, Panuzio e DiLillo (2010) investigaram casais recém-casados e os resultados evidenciaram que as mulheres exerciam mais violência psicológica e violência física do que os homens. Por fim, outras pesquisas revelam resultados e levantam discussões acerca da bidirecionalidade da violência conjugal, ou seja, a violência cometida por ambos os parceiros (Langhinrichsen-Rohling, 2010; Johnson, 2011; Straus, 2011).

Tendo em vista a complexidade relacional envolvida na conjugalidade, bem como a multiplicidade de fatores envolvidos enquanto desencadeadores e mantenedores de situações de violência (Guimarães e Silva, Valadares, \& Souza, 2013), é proeminente pensar e pesquisar as diversas formas e modalidades com as quais a violência pode ser evidenciada. Com intuito de contribuir acerca dessa discussão, Johnson (2006) descreve três subtipos de violência conjugal: terrorismo íntimo, resistência à violência e violência situacional. Os dois primeiros normalmente são derivados de estudos com populações clínicas, sendo a violência exercida por intermédio do controle e do poder em longo prazo, com intensidade grave e os homens como 
agressores mais frequentes. Por outro lado, a violência situacional é evidenciada na população em geral, sendo menos severa e normalmente bidirecional.

Considerando as variáveis sociodemográficas, estudos apontam associação da violência conjugal com idade, duração do relacionamento, filhos, nível de escolaridade, renda (Colossi et al., 2015; Kronbauer \& Meneghel, 2005; Vatnar \& Bjokly, 2012), não trabalhar fora (Lamoglia \& Manayo, 2009), abuso de álcool e outras drogas (Cunradi, Todd, \& Mair, 2015, Barros, 2016), ter sido vítima ou testemunha de violência na infância (Marasca, Colossi, \& Falcke, 2013), não ser casado oficialmente e não ser praticante de religião (Colossi et al., 2015).

Partindo de uma perspectiva sistêmica, o objetivo deste trabalho foi mapear as relações conjugais no Rio Grande do Sul (RS) e identificar a prevalência dos diferentes tipos de violência (violência física, agressão psicológica e coerção sexual) entre homens e mulheres. Além disso, buscou-se avaliar o poder preditivo das características sociodemográficas dos participantes para a ocorrência de violência conjugal.

\section{Método}

\section{Delineamento}

Trata-se de uma investigação quantitativa com delineamento descritivo e explicativo (Sampieri, Collado, \& Lucio, 2013).

\section{Participantes}

Participaram 751 casais que estavam em um relacionamento estável, de diferentes níveis socioeconômicos e de 67 municípios gaúchos, sendo 23 na região metropolitana e 44 cidades do interior do RS. Como critérios de inclusão, foi considerado estar casado ou em união estável há, no mínimo, seis meses. A coleta foi realizada através do mapeamento das diferentes regiões do Estado. Os casais foram contatados por conveniência, sendo solicitado que indicassem outros casais para participar do estudo, o que consistiu uma amostragem por "bola de neve".

As idades variaram entre 18 e 80 anos $(m=40,88 /$ $d p=11,09)$. Em relação à situação conjugal, $31 \%$ dos casais moravam juntos e $69 \%$ eram casados oficialmente. O tempo de união destes casais era de 4 meses a 51 anos $(m=15$ anos $/ d p=10,42$ anos $)$. A renda dos mesmos predominou de 1 a 3 salários mínimos, sendo que se obteve $49,4 \%$ das mulheres com esta renda e $37,8 \%$ dos homens. Em seguida, prevaleceu a renda de 4 a 6 salários mínimos, sendo $18,8 \%$ das mulheres e $27,6 \%$ dos homens.
TABELA 1

Informações gerais dos participantes.

\begin{tabular}{llc}
\hline Idade & $40,88(m)$ & $11,088(\mathrm{dp})$ \\
Tempo de relacionamento & 15,76 anos $(m)$ & 10,41 anos $(\mathrm{dp})$ \\
Situação conjugal & Casados oficialmente & $68,9 \%$ \\
& Morando juntos & $31,1 \%$ \\
Filhos & Tem & $78,8 \%$ \\
Escolaridade & Não tem & $21,1 \%$ \\
& Sem instrução formal & $9,4 \%$ \\
& Fundamental & $13,6 \%$ \\
Religião & Médio & $39,3 \%$ \\
& Superior & $37,6 \%$ \\
& Católica & $66 \%$ \\
& Evangélico & $13,3 \%$ \\
& Espírita & $7,4 \%$ \\
& Protestante & $3,5 \%$ \\
& Sem religião & $3,6 \%$ \\
& Outra & $6,3 \%$ \\
& Exerce atividade & $80,4 \%$ \\
& remunerada & \\
& Não exerce atividade & $19,6 \%$ \\
& remunerada & \\
\hline
\end{tabular}

$m=$ média; $d p=$ desvio padrão.

\section{Instrumentos}

Levantamento de Dados Sociodemográficos: destinado a investigar aspectos como a idade, situação conjugal, escolaridade, renda, se já fez terapia e religião, assim como informação sobre os filhos.

Revised Conflict Tactics Scales (CTS2): adaptada para o português por Moraes, Hasselmann e Reichenheim (2002) contém, no total, 78 itens. Estes formam cinco escalas que representam as seguintes dimensões: 1) violência física; 2) agressão psicológica; 3) coerção sexual; 4) lesão corporal; 5) negociação. A escala foi adaptada, neste estudo, para que o sujeito respondesse de forma autoaplicável. No presente estudo, foram utilizadas as três primeiras dimensões e o ponto de corte para considerar presença e ausência de violência foi resposta positiva para a ocorrência de cada uma delas em algum momento do relacionamento. Considerando a violência física, Straus (1995) define tanto as agressões consideradas como menores (empurrar, agarrar, dar tapa, jogar objeto, entre outros) quanto as graves (dar soco, queimar ou escaldar, usar uma faca ou arma de fogo, entre outros). A agressão psicológica é denominada como menor (gritos, ofensas, ameaças, entre outros) ou grave (desqualificar o desempenho sexual do outro, destruir objeto pessoal do outro, entre outros). Coerção sexual menor refere-se à insistência em fazer sexo sem utilização de força física, ou obrigar a ter relações sem preser- 
vativo, enquanto que a coerção sexual grave corresponde a ações de ameaças ou utilização de força e armas para coagir o parceiro a fazer sexo. No estudo de adaptação para o Brasil, não foram apresentados os índices de consistência interna. No presente estudo, o alpha de Cronbach obtido foi 0,819 pra a escala total.

\section{Procedimentos de coleta de dados}

Esta pesquisa faz parte de uma pesquisa maior, intitulada "Mapeamento e intervenção nas relações conjugais no RS: questões de gênero, resolução de conflitos e violência", que tem como colaboradores seis universidades do estado do Rio Grande do Sul (Edital PRONEX - CNPq e Fapergs). O projeto principal foi submetido ao Comitê de Ética da UFRGS, tendo sido aprovado (parecer $n^{\circ}$ 2010/011). A equipe de pesquisa era constituída por professores de diversas universidades e alunos de graduação, mestrado e doutorado. A coleta de dados foi realizada em todo o Estado através desses colaboradores. A partir do mapeamento das diferentes regiões do Rio Grande do Sul e da definição do número de casais necessários de cada região para compor uma amostra representativa do Estado, os casais foram selecionados por conveniência através dos contatos dos pesquisadores. Foi marcado um encontro com o casal para aplicação dos questionários, sendo entregue um envelope para cada cônjuge, contendo os questionários e os Termos de Consentimento Livre e Esclarecido. Foi explicada a pesquisa e os participantes assinaram o termo. Os membros do casal respondiam separadamente ao instrumento que era lacrado em um envelope na frente dos sujeitos para que tivessem assegurado o sigilo dos dados.

\section{Procedimentos de análise dos dados}

Foram realizadas estatísticas descritivas para verificar o comportamento das variáveis, através do SPSS 20.0. A análise da associação entre idade, tempo de casamento e violência foi feita através da Correlação de Pearson. A comparação das médias dos índices de violência conforme os níveis de escolaridade e renda foram analisadas através do teste ANOVA, e a comparação conforme situação conjugal e ter feito ou não psicoterapia foi realizada através do teste $t$. Por fim, análises bivariadas e ajustadas testaram a associação entre dados sociodemográficos como preditores de violência física e de violência psicológica. A medida de associação empregada foi o risco relativo (RR), assumindo-se intervalos de confiança em nível de $95 \%$, obtidos através de equações de estimação generalizadas (Zeger, Liang, \& Albert, 1988). Para seleção do modelo final, foram incluídas todas as variáveis sociodemográficas com valor de $p<.02$ na análise bivariada (Victoria, Huttly, Fuchs, \& Olinto, 1997).

\section{Resultados}

Os resultados da prevalência dos tipos de violência conjugal cometidos pelos membros do casal, homens e mulheres, podem ser analisados na Figura 1.

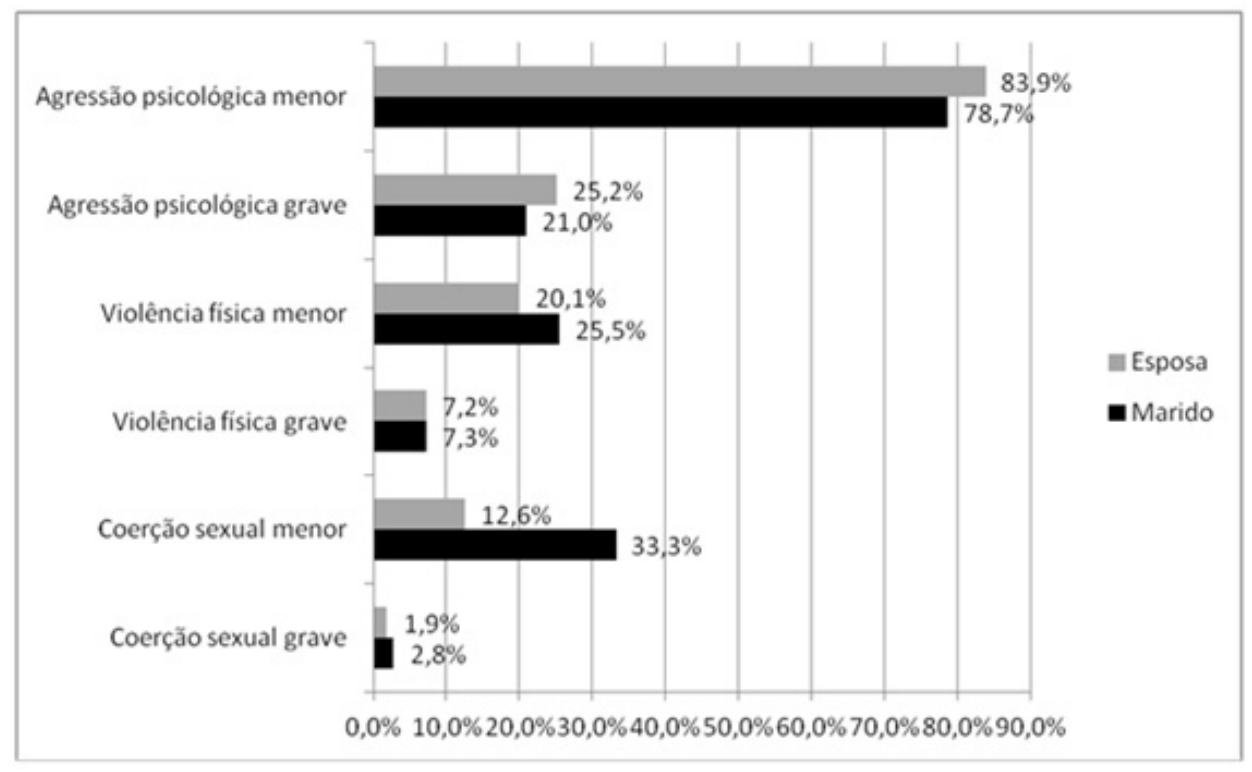

Figura 1. Porcentagem dos tipos de violência dos participantes, conforme o sexo. 
Observa-se que os índices de violência cometidos pelos sujeitos variaram de $1,9 \%$ de mulheres que revelaram ter cometido coerção sexual grave até $83,9 \%$ de mulheres que reconheceram terem cometido violência psicológica menor. Na comparação entre os sexos, verifica-se que houve diferença significativa na coerção sexual menor $(p<0,001)$, que foi mais cometida pelos homens $(m=3,75)$ do que pelas mulheres $(m=3,26)$, na agressão psicológica grave $(p=0,017)$, que foi mais cometida pelas mulheres $(m=3,47)$ do que pelos homens $(m=3,36)$ e na agressão psicológica menor $(p<0,001)$, também mais cometida pelas mulheres $(m=8,28)$ do que pelos homens $(m=7,84)$. Não foram observadas diferenças significativas nos dados referentes às dimensões de violência física.

Buscando analisar o perfil de casais em situação de violência, foram analisadas as associações entre os dados obtidos pela CTS2 e os dados sociodemográficos. Considerando a variável idade, a análise de Correlação de Pearson evidenciou correlação com: Violência Física Menor $(r=-0,104 ; p<0,001)$ e Agressão Psicológica Menor $(r=-0,083 ; p=0,001)$. Os resultados revelaram que quanto menor a idade do sujeito, maiores foram os índices desses tipos de violência. A comparação entre os participantes que já fizeram psicoterapia com os que não fizeram evidenciou diferença significativa nas dimensões de agressão psicológica menor $(t=-3,583$; $p<0,01)$ e coerção sexual grave $(t=2,334 ; p=0,02)$, sendo que o grupo que já fez psicoterapia apresentou média superior de agressão psicológica menor $(8,47)$ em comparação aos que não fizeram $(7,91)$, mas média inferior de coerção sexual grave $(4,02)$ em comparação aos que não fizeram $(4,07)$.

Através da ANOVA, verificou-se que a maioria dos tipos de violência apresentou índices diferenciados conforme a escolaridade dos sujeitos: Coerção sexual grave $(F=2,644 ; p=0,048)$, Coerção sexual menor $(F=4,461 ; p=0,04)$; Violência Física grave $(F=4,537$; $p=0,004)$ e Violência Física Menor $(F=5,992$; $p<0,001)$. Observaram-se diferenças entre os níveis de escolaridade, sendo que o maior nível correspondeu a menor ocorrência de violência.

Para verificar diferenças entre as médias de renda com a violência, também foi usada a ANOVA já que a renda foi questionada através de intervalos de salários mínimos. Observou-se diferença entre os grupos organizados conforme a renda nos seguintes tipos de violência: Violência Física Menor $(F=3,986$; $p=0,003)$; Agressão Psicológica Grave $(F=8,437$; $p<0,001)$ e Agressão Psicológica Menor $(F=6,451$; $p<0,001)$. Constatou-se que os maiores índices de violência foram obtidos pelos participantes com menor renda (até três salários mínimos) e pelos participantes com renda superior a dez salários mínimos. Dessa forma, percebeu-se, através das análises realizadas, que os participantes dos grupos com renda intermediária foram os que apresentaram os menores índices de violência.

Considerando as variáveis concernentes ao relacionamento conjugal, verificou-se que o tempo de relacionamento também se correlacionou com Violência Física Menor $(r=-0,064 ; p=0,04)$, indicando que quanto menor o tempo de casamento dos sujeitos, maiores foram os índices de violência. Em relação à situação conjugal, por intermédio do teste $t$, verificaram-se diferenças significativas no que tange aos índices mais elevados de violência nos sujeitos que moram juntos quando comparados aos que são casados oficialmente, nos seguintes tipos de violência: Coerção Sexual Menor $(t=-2,245 ; p=0,029)$ e Violência Física Menor $(t=-2,991 ; p<0,001)$.

No que diz respeito às variáveis sociodemográficas como preditoras da violência física, psicológica e sexual, foram realizadas equações de estimação generalizadas. Os resultados obtidos estão descritos na Tabela 2, adiante.

Conforme se observa na Tabela 2, verificou-se que estar casado oficialmente foi um fator de proteção $(p=0,002)$ em relação à chance de cometer violência física; enquanto que os fatores de risco foram: ser pouco praticante de religião $(p=0,002)$, ser homem $(p=0,014)$, ser mais jovem $(p<0,001)$ e ter pouco tempo de relacionamento $(p=0,012)$.

Quanto à perpetração de agressão psicológica, observaram-se como fatores de risco: estar na primeira união conjugal $(p=0,027)$, não ter ensino fundamental incompleto $(p<0,001)$, ter renda de 1 a 3 salários mínimos $(p=0,030)$, ser pouco praticante de religião $(p=0,027)$, residir com filhos $(p=0,011)$, ser mulher $(p<0,001)$, ser mais jovem $(p=0,045)$. Por outro lado, ter renda de 4 a 6 salários mínimos mostrou-se como fator de proteção quanto à perpetração da violência psicológica $(p=0,032)$.

Os fatores de risco para praticar coerção sexual são: ser homem $(p<0,01)$ e não ser praticante de religião $(p<0,01)$. Os fatores de proteção são: estar casado oficialmente $(p=0,05)$, não exercer atividade remunerada $(p=0,01)$ e não ter renda pessoal $(p=0,03)$. 
TABELA 2

Preditores de violência física, psicológica e sexual

\begin{tabular}{|c|c|c|c|c|c|c|c|c|c|c|c|c|}
\hline & \multicolumn{4}{|c|}{ Violência Física } & \multicolumn{4}{|c|}{ Agressão Psicológica } & \multicolumn{4}{|c|}{ Coerção sexual } \\
\hline & $R R$ & \multicolumn{2}{|c|}{ IC95\% } & $P$ & $R R$ & \multicolumn{2}{|c|}{ IC95\% } & $P$ & $R R$ & \multicolumn{2}{|c|}{ IC95\% } & $P$ \\
\hline Casado & 0,70 & 0,56 & 0,88 & 0,98 & 0,98 & 0,91 & 1,06 & 0,669 & 0,82 & 0,67 & 1,00 & 0,055 \\
\hline União estável & 1,00 & - & - & 1,00 & 1,00 & - & - & - & 1,00 & - & - & - \\
\hline \multicolumn{13}{|l|}{ Relação anterior } \\
\hline Não & 1,02 & 0,77 & 1,36 & 1,13 & 1,13 & 1,01 & 1,25 & 0,027 & 1,21 & 0,90 & 1,63 & 0,199 \\
\hline Sim & 1,00 & - & - & 1,00 & 1,00 & - & - & - & 1,00 & - & - & - \\
\hline \multicolumn{13}{|l|}{ Escolaridade } \\
\hline Fundamental incompleto & 1,11 & 0,75 & 1,65 & 1,25 & 1,25 & 1,10 & 1,41 & $<0,001$ & 1,31 & 0,90 & 1,90 & 0,165 \\
\hline Fundamental & 1,34 & 0,99 & 1,80 & 1,10 & 1,10 & 0,97 & 1,24 & 0,123 & 0,95 & 0,66 & 1,38 & 0,800 \\
\hline Médio & 0,93 & 0,71 & 1,20 & 1,09 & 1,09 & 0,99 & 1,19 & 0,091 & 1,28 & 0,98 & 1,67 & 0,070 \\
\hline Superior & 0,85 & 0,62 & 1,16 & 1,04 & 1,04 & 0,94 & 1,15 & 0,437 & 1,13 & 0,83 & 1,54 & 0,435 \\
\hline Pós-Graduação & 1,00 & - & - & 1,00 & 1,00 & - & - & - & 1,00 & - & - & - \\
\hline \multicolumn{13}{|l|}{ Trabalho } \\
\hline Não & 0,86 & 0,68 & 1,09 & 0,97 & 0,97 & 0,89 & 1,05 & 0,415 & 0,69 & 0,52 & 0,91 & 0,010 \\
\hline Sim & 1,00 & - & - & 1,00 & 1,00 & - & - & - & 1,00 & - & - & - \\
\hline \multicolumn{13}{|l|}{ Renda (salário) } \\
\hline Sem renda & 0,78 & 0,52 & 1,18 & 1,08 & 1,08 & 0,96 & 1,21 & 0,208 & 0,64 & 0,43 & 0,96 & 0,032 \\
\hline 1 a 3 & 1,20 & 0,91 & 1,58 & 1,11 & 1,11 & 1,01 & 1,21 & 0,030 & 0,84 & 0,65 & 1,08 & 0,181 \\
\hline 4 a 6 & 0,98 & 0,72 & 1,33 & 0,89 & 0,89 & 0,80 & 0,99 & 0,032 & 1,05 & 0,80 & 1,37 & 0,727 \\
\hline 7 a 9 & 0,77 & 0,50 & 1,19 & 0,94 & 0,94 & 0,82 & 1,07 & 0,349 & 0,73 & 0,48 & 1,09 & 0,122 \\
\hline 10 ou mais & 1,00 & - & - & 1,00 & 1,00 & - & - & - & 1,00 & - & - & - \\
\hline \multicolumn{13}{|l|}{ Psicoterapia } \\
\hline Não & 0,84 & 0,69 & 1,03 & 0,96 & 0,96 & 0,89 & 1,03 & 0,261 & 1,21 & 0,97 & 1,52 & 0,090 \\
\hline Sim & 1,00 & - & - & 1,00 & 1,00 & - & - & - & 1,00 & - & - & - \\
\hline \multicolumn{13}{|l|}{ Religiosidade } \\
\hline Nada & 1,10 & 0,74 & 1,64 & 1,04 & 1,04 & 0,92 & 1,18 & 0,555 & 1,72 & 1,20 & 2,45 & 0,003 \\
\hline Pouco & 1,60 & 1,19 & 2,15 & 1,12 & 1,12 & 1,01 & 1,24 & 0,027 & 1,30 & 0,95 & 1,77 & 0,097 \\
\hline Médio & 1,27 & 0,95 & 1,70 & 1,11 & 1,11 & 1,01 & 1,22 & 0,033 & 1,29 & 0,95 & 1,75 & 0,104 \\
\hline Muito & 1,00 & - & - & 1,00 & 1,00 & - & - & - & 1,00 & - & - & - \\
\hline \multicolumn{13}{|l|}{ Filhos } \\
\hline Sem filhos & 1,05 & 0,81 & 1,35 & 0,95 & 0,95 & 0,87 & 1,03 & 0,194 & 1,10 & 0,88 & 1,37 & 0,399 \\
\hline Com filhos & 1,00 & - & - & 1,00 & 1,00 & - & - & - & 1,00 & - & - & - \\
\hline \multicolumn{13}{|l|}{ Reside com filhos } \\
\hline Não & 0,80 & 0,61 & 1,05 & 0,90 & 0,90 & 0,83 & 0,98 & 0,011 & 0,94 & 0,76 & 1,17 & 0,599 \\
\hline Sim & 1,00 & - & - & 1,00 & 1,00 & - & - & - & 1,00 & - & - & - \\
\hline \multicolumn{13}{|l|}{ Cônjuge } \\
\hline Homem & 1,20 & 1,04 & 1,39 & 0,91 & 0,91 & 0,87 & 0,96 & $<0,001$ & 2,46 & 2,00 & 3,03 & 0,000 \\
\hline Mulher & 1,00 & - & - & 1,00 & 1,00 & - & - & - & 1,00 & - & - & - \\
\hline Idade & 0,981 & 0,972 & 0,990 & 0,997 & 0,997 & 0,993 & 1,000 & 0,045 & 0,993 & 0,985 & 1,002 & 0,123 \\
\hline Tempo de casamento & 0,999 & 0,998 & 1,000 & 1,000 & 1,000 & 1,000 & 1,000 & 0,235 & 0,999 & 0,999 & 1,000 & 0,082 \\
\hline
\end{tabular}




\section{Discussão}

Considerando tratar-se de uma população não clínica, os índices de violência, que variaram de 1,9\% para coerção sexual grave cometida pelas mulheres até $83,9 \%$ de violência psicológica menor também cometida por elas, evidenciam que a violência está presente em muitos relacionamentos conjugais, sob diferentes formas. Deve-se atentar ainda ao fato de que é comum a ocorrência concomitante dos diversos tipos de violência (Panuzio \& DiLillo, 2010; Schraiber et al., 2007).

A violência conjugal é um fenômeno complexo, com múltiplas formas de expressão, que, muitas vezes, não chega a ser denunciado, pela dificuldade dos cônjuges em reconhecer suas interações como violentas (Garcia et al., 2008), especialmente nos casos de agressão psicológica. Esse aspecto parece ser muito significativo, pois a primeira possibilidade de enfrentar o problema passa pelo seu reconhecimento, ou seja, superar as barreiras que tornam a violência conjugal um fenômeno indecifrável (Gomes e al., 2012; Monteiro \& Souza, 2007).

No presente estudo, ao comparar-se a violência cometida pelos cônjuges, homens e mulheres, verificou-se que a coerção sexual menor foi significativamente mais cometida por homens do que por mulheres, enquanto que a violência psicológica grave e menor foi significativamente mais cometida por mulheres. Esses resultados vão ao encontro de estudos prévios (Colossi et al., 2015; Gomes, 2003) a respeito da predominância dos homens como autores de agressão sexual e das mulheres como autoras de violência psicológica. Em relação à violência física grave ou menor, verificou-se que não houve diferença significativa na violência cometida por homens e mulheres, o que pode estar confirmando estudos com população não clínica que evidenciam maior mutualidade e simetria nesse tipo de violência (Straus, 2011). Este resultado revela a necessidade de atentarmos para o risco de uma visão estereotipada do homem como agressor e da mulher como vítima, presente de forma exclusiva na elaboração de políticas públicas. Inclusive o risco de, como profissionais da saúde, estarmos produzindo violência ao ouvir um dos membros do casal somente como agressor e o outro somente como vítima (Alvim \& Souza, 2005). É possível que os homens tenham maior dificuldade de denunciar a violência que sofrem em seus relacionamentos, considerando os tradicionais estereótipos de gênero, que associam masculinidade à força e virilidade física (Falcke, Oliveira, Rosa, \& Bentancur, 2009).

Em relação à variável idade e ao tempo de casamento, identificou-se que houve uma correla- ção negativa com dimensões da violência. Esse resultado aponta para uma maior intensidade dos relacionamentos recentes, de pessoas mais jovens, que seriam caracterizados como mais violentos. Em termos de ciclo de vida conjugal, pode-se pensar que as etapas iniciais do relacionamento são caracterizadas pela necessidade de um maior ajuste e do estabelecimento de uma conjugalidade que integre os modelos de relacionamentos de cada um dos cônjuges trazidos de sua família de origem, o que demanda negociação e pode resultar em violência quando as estratégias de resolução de conflitos não são funcionais (Carter \& McGoldrick, 2005; Marasca, Colossi, \& Falcke, 2013).

A escolaridade foi uma variável que se correlacionou negativamente com a violência, indicando que quanto maior a escolaridade do casal, menor é a violência. $\mathrm{Na}$ medida em que as pessoas vão ampliando a sua escolaridade, observa-se uma maior possibilidade de argumentação, o que se reflete em maior capacidade de negociação, e consequentemente, menor índice de violência. Nesse sentido, participantes com maiores níveis de escolaridade costumam demonstrar formas mais saudáveis para resolução dos seus conflitos (Mosmann \& Falcke, 2011; Gomes et al., 2012).

Considerando a renda, identificou-se que os casais com menor renda (menos de 3 salários mínimos) ou com maior renda (mais de 10 salários mínimos) apresentaram os maiores índices de violência. Especificamente no que tange aos casais com maior renda, o presente estudo contraria pesquisas anteriores que descrevem uma correlação negativa entre renda e violência (Amaral et al., 2016; Kronbauer \& Meneghel, 2005). Evidencia-se que a violência se faz presente em diferentes níveis socioeconômicos, ainda que possivelmente seja mais denunciada em níveis socioeconômicos menos favorecidos, o que leva a uma percepção preconcebida de sua prevalência em populações com menor renda. Podem estar implicados nesses resultados diferentes aspectos contextuais, tais como facilidade de acesso a serviços, a profissionais e a informações, que podem estar mais disponíveis para os níveis socioeconômicos médios.

Pessoas que já fizeram psicoterapia apresentaram menores índices de coerção sexual grave, mas maiores índices de agressão psicológica menor. Considerando que não se avaliou o motivo de busca pelo tratamento, tampouco os índices de violência pré e pós psicoterapia, ou seja, o possível impacto da mesma na violência, a análise desses resultados mostra-se um tanto restrita. Ainda assim, pode-se pensar que pessoas com maiores níveis de violência sexual nem cheguem à psicoterapia ou que essa tenha auxiliado na contenção desse tipo de violência. Da mesma forma, pode-se inferir que 
as pessoas que fizerem psicoterapia e apresentaram maiores índices de violência psicológica menor poderiam, antes do processo psicoterápico, apresentar maiores índices ainda. São dados intrigantes, que merecem maiores investimentos de estudos futuros.

Dentre as variáveis do relacionamento conjugal, além do tempo de casamento, observou-se que houve diferença significativa nos índices de violência de casais que são casados oficialmente quando comparados aos casais que moram junto. Os casais que moram juntos apresentaram níveis mais elevados de coerção sexual menor e violência física menor. Esse dado evidencia que a oficialização do matrimônio pode ser considerada fator protetivo em relação à violência conjugal. A oficialização do casamento pode ser compreendida como um dos diversos ritos de passagem, o qual auxilia na transição do ciclo de vida familiar (Carter \& McGoldrick, 2005). Nesse processo, tanto o casal quando às famílias de origem experienciam processos de readaptação à nova fase, fator que pode favorecer a busca por auxílio frente aos conflitos conjugais cotidianos.

Os dados obtidos por meio de equações de estimação generalizadas permitiram identificar variáveis sociodemográficas que se caracterizaram como risco ou proteção para cometer os diferentes tipos de violência. Em relação à violência física, constatou-se que os participantes homens, mais jovens, que coabitavam com as esposas e eram pouco praticantes de religião tinham mais chances de perpetrá-la. $\mathrm{O}$ fato de ser mulher, jovem, em primeira união, sem instrução formal, com baixa renda e pouco praticante de religião aumentava as chances de cometer agressão psicológica. Por fim, quanto à coerção sexual, ser homem, em união estável, não praticante de religião, com trabalho remunerado e maior renda ampliava as chances de perpetração desse tipo de violência. Estes resultados não indicam necessariamente um perfil de perpetradores, mas características que aumentam as chances de cometer os diferentes tipos de violência. Chama a atenção, além das variáveis demográficas já analisadas, os perfis envolverem o nível de prática religiosa. O resultado corrobora estudos prévios (Colossi et al., 2015; Cunradi, Caetano, \& Schafer, 2002), nos quais a prática religiosa esteve associada a menores índices de violência. Curandi, Caetano e Schafer (2002), no entanto, destacam que a religião não teve uma forte influência direta na ocorrência de violência, mas foi um fator de mediação, que pode ter um importante papel em intervenções primárias e secundárias de prevenção da violência conjugal.

\section{Considerações finais}

O presente estudo permitiu o mapeamento da violência em casais do Rio Grande do Sul e a identificação de variáveis sociodemográficas que se mostraram associadas à sua ocorrência. Os achados remetem a existência de agressões mútuas entre os casais, diferentemente do que apontam os tradicionais estudos de gênero. Esse dado insere um questionamento sobre como as políticas públicas e os atuais serviços de saúde estão compreendendo, acolhendo os envolvidos e intervindo nas situações de violência conjugal. Destaca-se assim que a proposta do estudo de investigar tanto a perpetração da violência pelos homens quanto pelas mulheres vem na direção de romper com uma visão centrada nos casais que configuram o terrorismo íntimo (Johnson, 2006, 2011). É evidente a relevância de tais pesquisas; no entanto, se faz necessário pesquisar outras formas de manifestação da violência conjugal, as quais homens e mulheres mostram-se como perpetradores e vítimas.

Considerando que os serviços dedicam-se preponderantemente ao acolhimento da vítima e punição do agressor, questiona-se que tipo de atenção está sendo despendida aos homens que se sentem vitimizados por suas parceiras ou mesmo às mulheres que utilizam a violência como tentativa de resolução de seus conflitos. Compreendendo que homens e mulheres podem assumir diferentes papéis no relacionamento conjugal, fugindo da dicotomia entre vítima e agressor, atenta-se para o fato de que os profissionais da saúde devem estar abertos e preparados para entender a diversidade de possibilidades de expressão da violência entre casais. Somente com esse olhar será possível favorecer com que todas as formas de violência sejam reconhecidas e denunciadas, contribuindo para a diminuição dos índices de subnotificação, pois, se os dados de violência contra a mulher muitas vezes não chegam a serem denunciados, os índices de violência contra o homem parecem ser ainda mais imprecisos. Nesse sentido, entende-se que a prevenção depende da atenção dada aos casais, do envolvimento dos serviços e da compreensão do fenômeno, incluindo a dinâmica das relações conjugais.

Levando em conta as demais variáveis sociodemográficas, identifica-se os casais com menores níveis de escolaridade, sem prática religiosa, nos anos iniciais do casamento e em coabitação sem oficialização como em risco para uma maior ocorrência de violência conjugal. Neste sentido, estratégias interventivas, tais como grupos psicoeducativos e protocolos de intervenção para casais, devem ser pensadas com a finalidade de 
favorecer com que os casais, especialmente os mais jovens, desenvolvam um contrato de relacionamento claro e coerente, que contemple estratégias construtivas de resolução de conflitos, envolvendo a escuta ativa, a busca pela compreensão e disponibilidade para chegar a um acordo através do diálogo.

\section{Agradecimentos}

Agradecemos à Larissa Wolff da Rosa (in memorian), pela colaboração na realização deste trabalho; e ao FAPERGS/CNPq, pelo apoio financeiro que viabilizou a conclusão e sua publicação.

\section{Referências}

Amaral, L., Vasconcelos, T., Sá, F., Silva, A., \& Macena, R. (2016). Violência doméstica e a Lei Maria da Penha: perfil das agressões sofridas por mulheres abrigadas em unidade social de proteção. Estudos Feministas, 24(2), 521-540. https://doi.org/10.1590/1805-9584-2016v24n2p521

Alvim, S. F., \& Souza, L. de (2005). Violência conjugal em uma perspectiva relacional: homens e mulheres agredidos/ agressores. Psicologia: Teoria e Prática, 7(2), 171-206.

Barros, E. N., Silva, M. A., Neto, G. H. F., Lucena, S. G., Ponzo, L. \& Pimentel, A. P. (2016). Prevalência e fatores associados à violência por parceiro íntimo em mulheres de uma comunidade em Recife/Pernambuco, Brasil. Ciência \& Saúde Coletiva, 21(2), 591-598. https://doi.org/10.1590/1413-81232015212.10672015

Carmo, R., Grams, A., \& Magalhães, T. (2011). Men as victims of intimate partner violence. Journal of Forensic and Legal Medicine, 18, 355-259. https://doi.org/10.1016/j.jflm.2011.07.006

Carter, B. \& McGoldrick, M. (1995). As mudanças no ciclo de vida familiar: Uma estrutura para terapia familiar. Porto Alegre: Artes Médicas.

Colossi, P. M., Razera, J., Haack, K. R., \& Falcke, D. (2015). Violência conjugal: Prevalência e fatores associados. Contextos Clínicos, 8(1), 55-66. https://doi.org/10.4013/ctc.2015.81.06

Cunradi, C. B., Caetano, R., \& Schafer, J. (2002). Religious affiliation, denominational homogamy, and intimate partner violence among US couples. Journal for the Scientific Study of Religion, 41(1), 139-151. https://doi.org/10.1111/14685906.00106

Cunradi, C.B., Todd, M., \& Mair, C. (2015). Discrepant patterns of heavy drinking, marijuana use, and smoking and intimate partner violence: Results from the California community health study of couples. Journal of Drug Education, 45(2), 73-95. https://doi.org/10.1177/0047237915608450

Drijber, B. C., Reijnders, U. J. L., \& Ceelen, M. (2013). Male victims of domestic violence, Journal of Family Violence, 28, 173-178. https://doi.org/10.1007/s10896-012-9482-9

Falcke, D., Oliveira, D. Z., Rosa, L. W., \& Bentancur, M. (2009). Violência conjugal: Um fenômeno interacional. Contextos Clínicos, 2(2), 81-90. https://doi.org/10.4013/ctc.2009.22.02

Garcia, M. V., Ribeiro, L. A., Jorge, M. T., Pereira, G. R., \& Resende, A. P. (2008). Caracterização dos casos de violência contra a mulher atendidos em três serviços na cidade de Uberlândia, Minas Gerais, Brasil. Cad. Saúde Pública, 24(11), 2551-2563. https://doi.org/10.1590/S0102-311X2008001100010

Gomes, R. A. (2003). Mulher em situação de violência sob a ótica da saúde. In M. C. Minayo \& E. R. Souza (Eds.). Violência sob o olhar da saúde: Infrapolitica da contemporaneidade brasileira (pp. 199-222). Rio de Janeiro, FIOCRUZ.

Gomes, N. P., Diniz, N. M. F., Camargo, L., \& Silva, N. P. (2012). Homens e mulheres em vivência de violência conjugal: Características socioeconômicas. Revista Gaúcha de Enfermagem, 33(2). https://doi.org/10.1590/S198314472012000200016

Guimarães e Silva, J., Valadares, F. C., \& Souza, E. R. de. (2013). O desafio de compreender a consequência fatal da violência em dois municípios brasileiros. Interface - Comunicação, Saúde, Educação, 17(46), 535-547. https://doi. org/10.1590/S1414-32832013005000022

Johnson, M. P. (2006). Conflict and control gender symmetry and asymmetry in domestic violence. Violence Against Women, 12(11), 1003-1018. https://doi.org/10.1177/1077801206293328

Johnson, M. P. (2011). Gender and types of intimate partner violence: A response to an anti-feminist literature review. Aggression and Violent Behavior, 16(4), 289-296. https://doi.org/10.1016/j.avb.2011.04.006

Kronbauer, J. F. D., \& Meneghel, S. N. (2005). Perfil da violência de gênero perpetrada por companheiro. Revista de Saúde Pública, 39(5), 695-701. https://doi.org/10.1590/S0034-89102005000500001

Lamoglia, C. V. A., \& Minayo, M. C. S. (2009). Violência conjugal, um problema social e de saúde pública: estudo em uma delegacia do interior do Estado do Rio de Janeiro. Ciência \& Saúde Coletiva, 14(2), 595-604. https://doi. org/10.1590/S1413-81232009000200028

Langhinrichsen-Rohling, J. (2010). Controversies involving gender and intimate partner violence in the United States. Sex Roles, 62(3-4), p. 179-193. https://doi.org/10.1007/s11199-009-9628-2

Leôncio, K. L., Baldo, P. L., João, V. M., \& Biffi, R. G. (2008). O perfil de mulheres vitimizadas e de seus agressores. Revista de Enfermagem, 16(3), 307-312. 
Linch, L. F. (2014). Observatório da Violência Contra as Mulheres: Uma trajetória, muitas histórias. In Comissão de Direitos Humanos e Cidadania. Relatório Lilás 2014 (pp. 106-131). Porto Alegre: Assembleia Legislativa do Rio Grande do Sul.

Lourenço, L. M., Baptista, M. N., Almeida, A. A., Basílio, C., Koga, B. M., Hashimoto, J. K. F., \& Andrade, G. C. (2013). Panorama da violência entre parceiros íntimos: Uma revisão crítica da literatura. Interamerican Journal of Psychology, 47(1), 91-99. Disponível em: http://www.redalyc.org/articulo.oa?id=28426980011

Lövestad, S. \& Krantz, G. (2012). Men's and women's exposure and perpetration of partner violence: an epidemiological study from Sweden, BioMed Central Public Health, 12, 945-955. https://doi.org/10.1186/1471-2458-12-945

Marasca, A. R., Colossi, P. M., \& Falcke, D. (2013). Violência conjugal e família de origem: Uma revisão sistemática da literatura de 2006 a 2011. Temas em Psicologia, 21(1), 221-243. https://doi.org/10.9788/TP2013.1-16

Monteiro, C. F. S. \& Souza, I. E. O. (2007). Vivência da violência conjugal: Fatos do cotidiano. Texto \& Contexto: Enfermagem, 16(1), 26-31. https://doi.org/10.1590/S0104-07072007000100003

Monteiro, M. F. G. \& Zaluar, A. (2012). Violência contra a mulher e a violação dos direitos humanos. Reprodução \& Climatério, 27(3), 91-97. https://doi.org/10.1016/j.recli.2012.11.001

Moraes, C. L., Hasselmann, M. H., \& Reichenheim, M. (2002). Adaptação transcultural para o português do instrumento "Revised Conflict Tactics Scales (CTS2)" utilizado para identificar violência entre casais. Cadernos de Saúde Pública, 18(1), 163-176. https://doi.org/10.1590/S0102-311X2002000100017

Mosmann, C. \& Falcke, D. (2011). Conflitos conjugais: Motivos e frequência. Revista da SPAGESP - Sociedade de Psicoterapias Analíticas Grupais do Estado de São Paulo, 12(2), 5-16.

Panuzio, J. \& DiLillo, D. (2010). Physical, psychological, and sexual intimate partner aggression among Newlywed couples: Longitudinal prediction of marital satisfaction. Journal of Family Violence, 25, 689-699. https://doi. org/10.1007/s10896-010-9328-2

Sampieri, R. H., Collado, C. F., \& Lucio, M. P. B. (2013). Metodologia de Pesquisa (5a ed.). São Paulo: Mc Graw Hill.

Schraiber, L. B. et al. (2007). Prevalência da violência contra a mulher por parceiro íntimo em regiões do Brasil. Revista de Saúde Pública, 41(5), 797-807. https://doi.org/10.1590/S0034-89102007000500014

Straus, M. A. (1995). Manual for the Conflict Tactics Scale. Durham, NH: Family Research Laboratory, University of New Hampshire.

Straus, M. A. (2011). Gender symmetry and mutuality in perpetration of clinical-level partner violence: Empirical evidence and implications for prevention and treatment. Aggression and Violent Behavior, 16(4), 279-288. https:// doi.org/10.1016/j.avb.2011.04.010

Vatnar, S. K. B. \& Bjokly, S. (2012). Does separation or divorce make any difference? An interactional perspective on intimate partner violence with focus on marital status. Journal of Family Violence, 27, 45-54. https://doi.org/10.1007/ s10896-011-9400-6

Victora, C. G., Huttly, S. R., Fuchs, S. C., \& Olinto, M. T. (1997). The role of conceptual frameworks in epidemiological analysis: A hierarchical approach. International Journal of Epidemiology, 26(1), 224-227. https://doi.org/10.1093/ ije/26.1.224

Waiselfisz, J. J. (2015). Mapa da Violência 2015: Homicídios de Mulheres no Brasil. FLACSO, Brasil. Disponível em: http://www.mapadaviolencia.org.br/pdf2015/MapaViolencia_2015_mulheres.pdf. Acesso em: 20 jun. 2016.

World Health Organization. (2013). Global and regional estimates of violence against women: prevalence and health effects of intimate partner violence and nonpartner sexual violence. Geneva: WHO Press.

Zeger, S. L., Liang, K. Y., \& Albert, P. S. (1988). Models for longitudinal data: A generalized estimating equation approach. Biometrics, 44(4), 1049-1060. https://doi.org/10.2307/2531734

Autores:

Denise Falcke - Doutora, Universidade do Vale do Rio dos Sinos.

Mariana Gonçalves Boeckel - Doutora, Universidade Federal de Ciências da Saúde de Porto Alegre.

Adriana Wagner - Pós-doutora, Universidade Federal do Rio Grande do Sul.

\section{Endereço para correspondência:}

Adriana Wagner

Instituto de Psicologia - UFRGS

Rua Ramiro Barcelos, 2600 - Santa Cecília

90035-003, Porto Alegre, RS, Brasil

<adrianawagner.ufrgs@hotmail.com>

Recebido em: 01.09.2016

Aceito em: 21.01.2017 